\title{
Aspectos Gerais e Práticos do EEG
}

\author{
Délrio Façanha da Silva* \\ Márcia Marques de Lima**
}

\section{RESUMO}

O conhecimento de alguns aspectos práticos na rotina da interpretação do EEG são importantes para evitarmos conclusões errôneas. Nas epilepsias, o uso correto das montagens facilita uma melhor interpretação do traçado e o EEG é imprescindível para o diagnóstico das síndromes. Nas demências, podemos encontrar diferentes padrões, sem nenhum achado patognomônico. Variantes benignas não devem ser interpretadas como anormalidades. Do mesmo modo, atividade epileptiforme no EEG, sem história de epilepsia, não justifica tratamento. O EEG do recém-nascido é um dos exames de maior importância diagnóstica e sobretudo prognóstica nas lesões encefálicas. Nos "status epilepticus" não-convulsivos, o EEG é fundamental para os diagnósticos diferenciais. Nas encefalopatias metabólicas, podemos avaliar o grau da encefalopatia pelo EEG. Nos comas, o EEG pode avaliar o prognóstico e confirmar o diagnóstico de morte encefálica. O mapeamento cerebral, exame realizado somente após a interpretação do EEG digital, não é um exame de rotina.

\section{UNITERMOS}

$\mathrm{EEG}$, aspectos gerais.
Médico Eletrencefalografista do Setor de EEG e Polissonografia da Disciplina de Neurologia - Universidade Federal de São Paulo - Escola Paulista de Medicina.

** Médica Eletrencefalografista do Setor de EEG e Polissonografia da Disciplina de Neurologia - Universidade Federal de São Paulo - Escola Paulista de Medicina.
Neste breve resumo geral a respeito de alguns assuntos dentro do vasto campo do EEG, abordaremos alguns aspectos práticos nas epilepsias, no recém-nascido, demências, encefalopatias metabólicas, nas variantes benignas e nos comas. Por último, resumidamente alguns conceitos de EEG digital $\mathrm{x}$ EEG convencional $\mathrm{x}$ mapeamento cerebral e sua aplicabilidade prática.

\section{EEG NAS EPILEPSIAS}

\section{Atividade epi leptiforme focal $x$ montagem}

Na dependência do tipo de montagem escolhido para a análise do EEG, a atividade epileptiforme focal (AEF) pode conduzir a uma errônea interpretação do traçado. Por exemplo, o foco temporal anterior (FTA) é bem evidenciado na montagem com referencial $\mathrm{Cz}$, assim como a onda aguda do vértex (OAV) (Fig. 1). Porém, podem ser interpretados de outra forma quando usarmos a montagem biauricular (Fig. 2): o FTA praticamente não é visualizado e passamos a observar uma onda aguda difusa e bilateral, com aspecto morfológico de "V", simulando a OAV (grafoelemento fisiológico do sono). Do mesmo modo, a figura 2 mostra logo ao lado do FTA, a nova apresentação da OAV que simula uma AEF. Em suma, o FTA e a OAV são bem visualizados na montagem com referencial $\mathrm{Cz}$, enquanto com a biauricular (Rf) o FTA simula a OAV e esta ao assumir um aspecto focal assemelha-se a uma AEF. Desse modo, na montagem com Rf (biauricular), devemos estar atentos para não interpretarmos o FTA como OAV, assim como a OAV como uma AEF. Isso decorre da contaminação do Rf pela FTA (pela proximidade de $\mathrm{F} 8$ ao $\mathrm{Rf}$ ) e pelos eletrodos centro-parietais serem $\mathrm{G} 1$ apenas em duas derivações à esquerda e à direita (C3-Rf, P3-Rf, C4-Rf, P4-Rf), enquanto na montagem com $\mathrm{Cz}$ não ocorreu a contaminação com o FTA e a $\mathrm{OAV}$ atinge todas as derivações por $\mathrm{Cz}$ ser $\mathrm{G} 2$.

A OAV é um achado normal no EEG durante o sono não-REM, porém devemos lembrar que temos os focos da linha média (FLM) e que estes são semelhantes às OAV e não devem ser confundidos com grafoelementos 


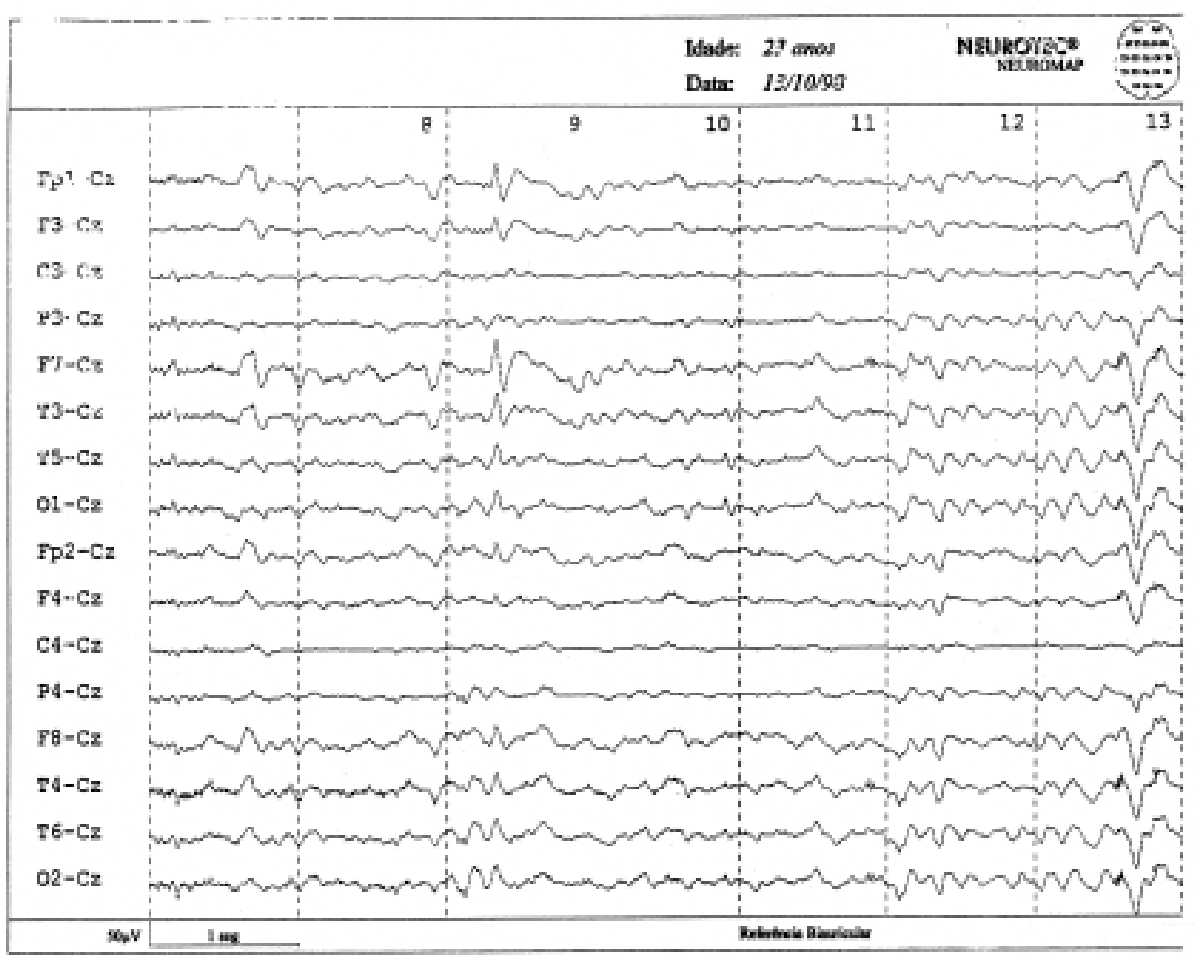

FIGURA 1

Presença de atividade epileptiforme de projeção temporal anterior esquerda, de fácil identificação na montagem com Cz.

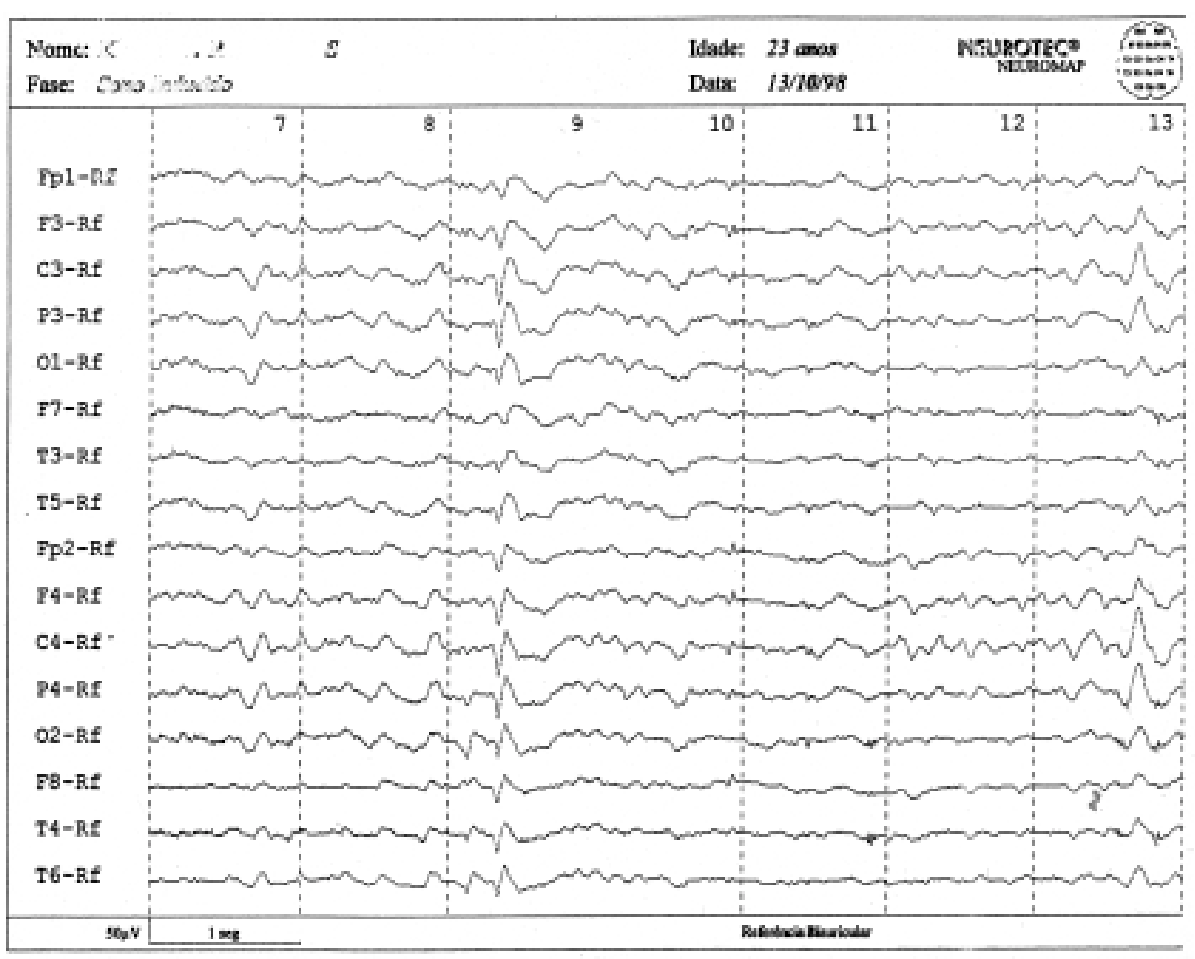

FIGURA 2

O mesmo momento da fig. 1, porém em montagem com eletrodo biauricular. fisiológicos. Do mesmo modo devemos estar atentos para não laudarmos as OAV como anormalidade. O diagnóstico diferencial pode ser feito pela presença de FLM durante a vigília, por polipontas e pela presença de dipolo tangencial ${ }^{1}$.

Outro cuidado é quando a AEF muda de lateralidade. A fig. 3 mostra claramente uma AEF esquerda com a utilização da montagem com referencial $\mathrm{Cz}$. Ao fazermos a mudança, no mesmo momento, para a montagem que utiliza o referencial Rf, a AEF é nitidamente melhor vizualizada no hemisfério cerebral direito (Fig. 4). As figs. 5 e 6 ilustram outras situações semelhantes. Nesses casos, a utilização de uma montagem transversa ou bipolar seqüencial pode ser útil na elucidação da real lateralidade. Isso tem muita importância na avaliação pré-cirúrgica de pacientes epilépticos.

A utilização de uma montagem bipolar seqüencial, sem a utilização de $\mathrm{Cz}$ ou de $\mathrm{Rf}$, pode não demonstrar a AEF ou apenas de forma frustrada devido à equipotencialidade, enquanto é de fácil identificação com referencial $\mathrm{Cz}$ no mesmo momento.

De maneira geral, inicialmente é recomendável uma análise de todo o traçado com a montagem referencial $\mathrm{Cz}$ e depois com $\mathrm{Rf}$ auricular (A1, A2 ou A1 + A2). As montagens bipolares seqüenciais e transversas utilizamos em um segundo tempo, após a prévia identificação da $\mathrm{AEF}$, e não como opção inicial de uma análise.

\section{Técnica}

O EEG de rotina em um paciente epiléptico, ou qualquer outro paciente deve ser realizado no mínimo por 30 minutos. Os eletrodos devem ser colocados corretamente de acordo com o sistema internacional 10$20^{2}$. O paciente deve estar em repouso, relaxado, bem alimentado, 


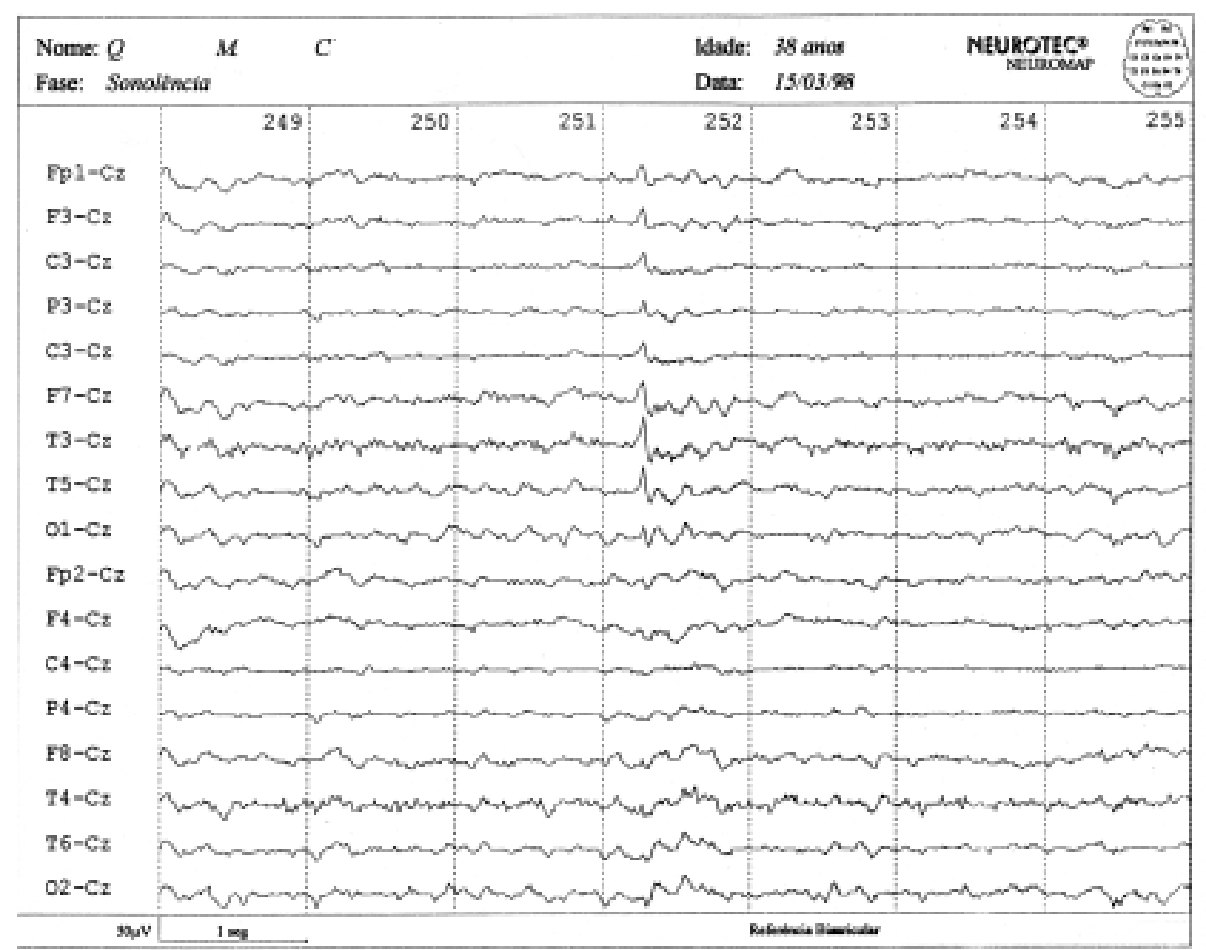

FIGURA 3

EEG mostra foco temporal esquerdo, em montagem com Cz.

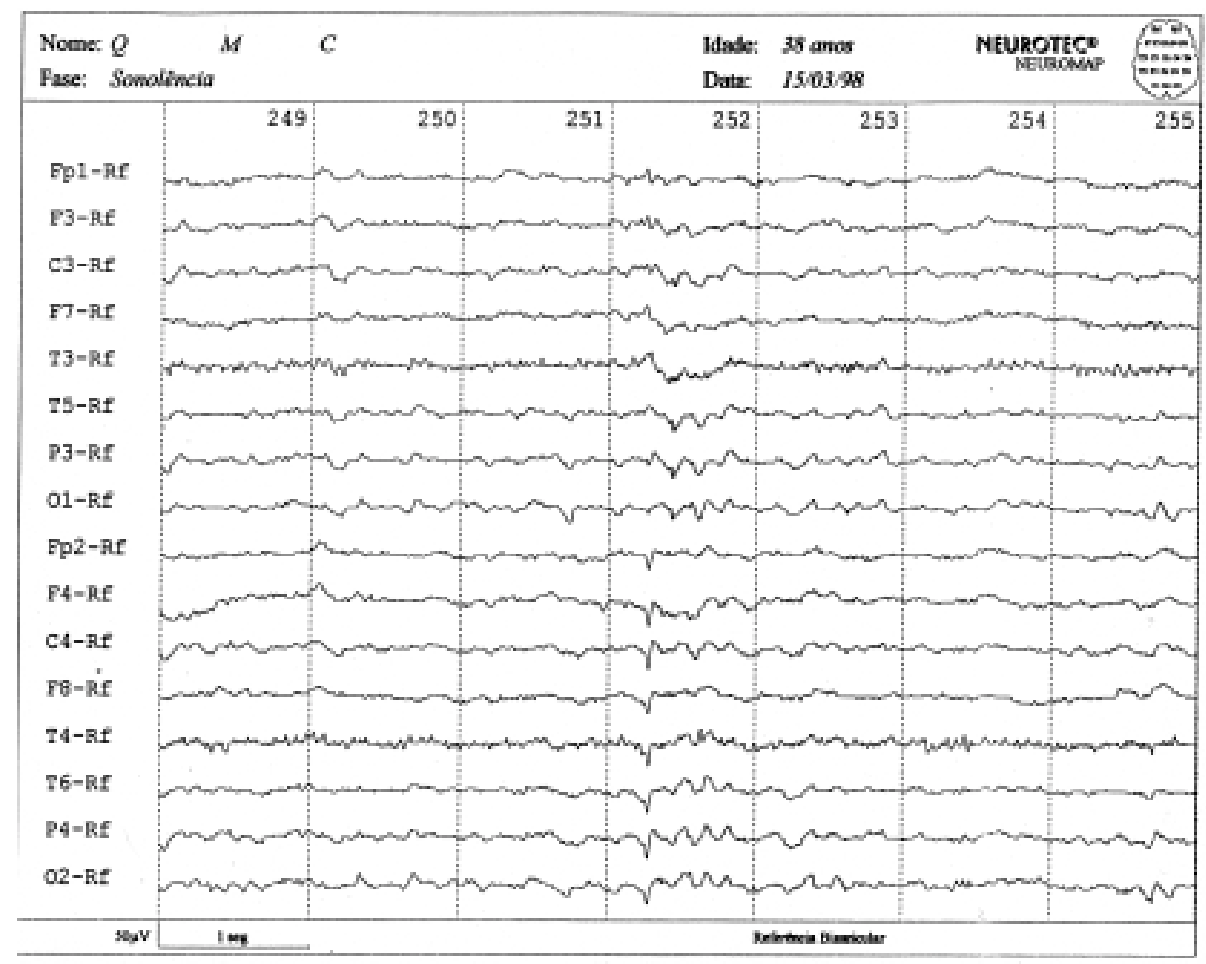

FIGURA 4

O foco temporal da fig. 3, no mesmo momento, agora melhor vizualizado à direita, em montagem com biauricular. com olhos fechados, em ambiente calmo e com a cabeça lavada e sem a utilização de cremes no couro cabeludo. Realizamos a hiperpnéia por 3 minutos, a fotoestimulação e a prova de abrir e fechar os olhos, para verificarmos a reatividade do ritmo alfa. Em crianças, é comum observarmos no EEG ondas lentas difusas e bilaterais (Fig. 7), frontais ou de predomínio posterior e que geralmente são normais. O EEG em sono é muito importante em todos os pacientes epilépticos.

Em relação ao sono, é imprescindível que o EEG seja realmente realizado em sono e que o paciente permaneça nesse estado por 30 minutos. A simples presença de fusos e ondas agudas do vértex por alguns segundos ou minutos, durante o traçado, não nos deve levar a escrevermos na conclusão do laudo que o exame foi realizado em sono, sobretudo se foi normal. Sabemos que nas fases III e IV do sono nãoREM (sono por ondas lentas) a sincronização é maior e, portanto, a possibilidade de obtermos uma atividade epileptiforme no EEG é muito grande. Se possível, a obtenção do sono REM, tem maior valor para localização da atividade epileptiforme.

\section{Crise de ausência ("Petit maladie")}

As crises de ausências (epilepsia generalizada primária) apresentam no EEG complexos ponta e polipontaonda a $3 \mathrm{~Hz}$, difusos e bilaterais, sobretudo durante a hiperpnéia, e atividade de base normal (Fig. 8). Entretanto, também podemos encontrar atividade lenta delta difusa e bilateral ${ }^{3}$, isoladamente, ou precedendo os complexos ponta-onda a $3 \mathrm{~Hz}$. A presença de AEF não descarta o diagnóstico de ausência, pois podemos encontrar no EEG desses pacientes, além dos clássicos complexos ponta-onda $3 \mathrm{~Hz}$, AEF na 


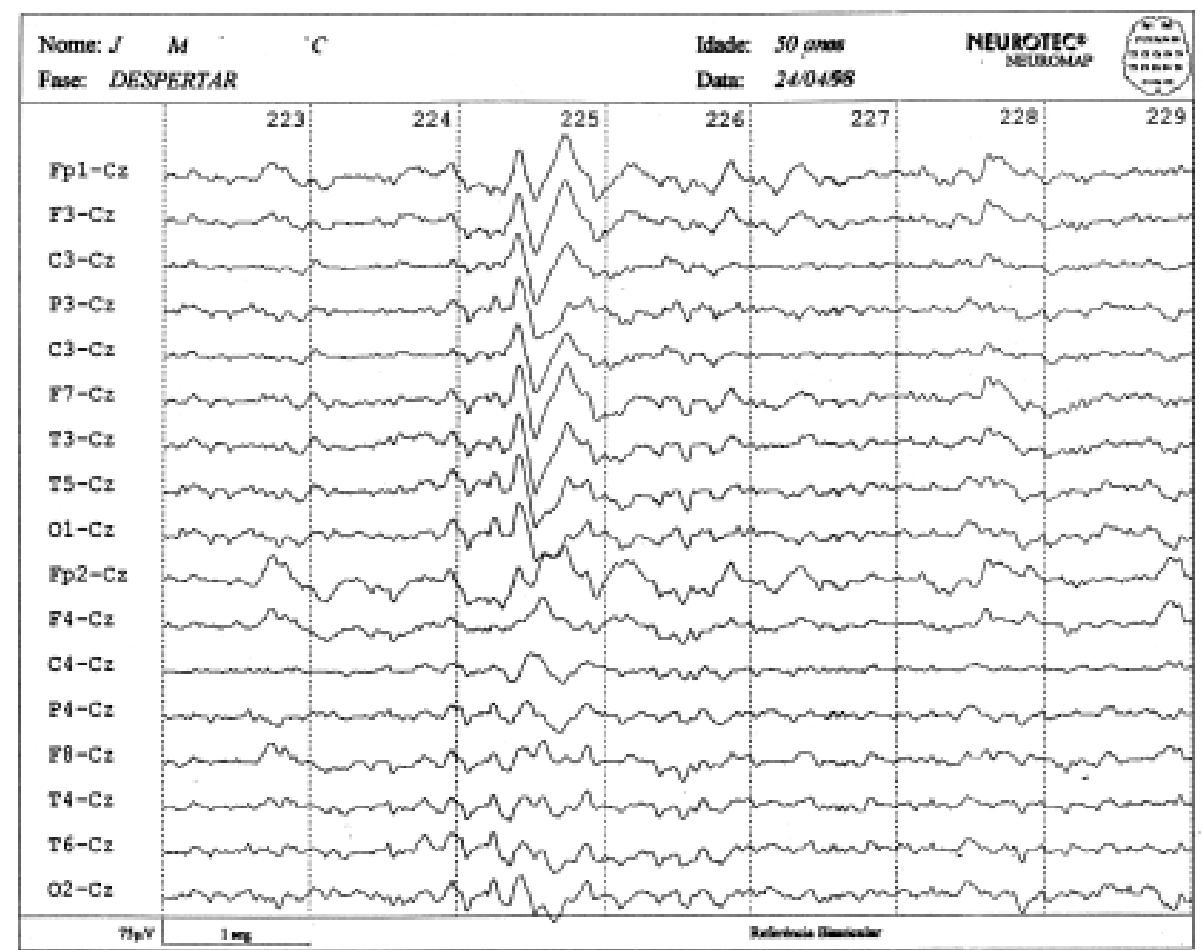

FIGURA 5

EEG evidencia atividade epileptiforme de projeção frontotemporal esquerda, em montagem com Cz.

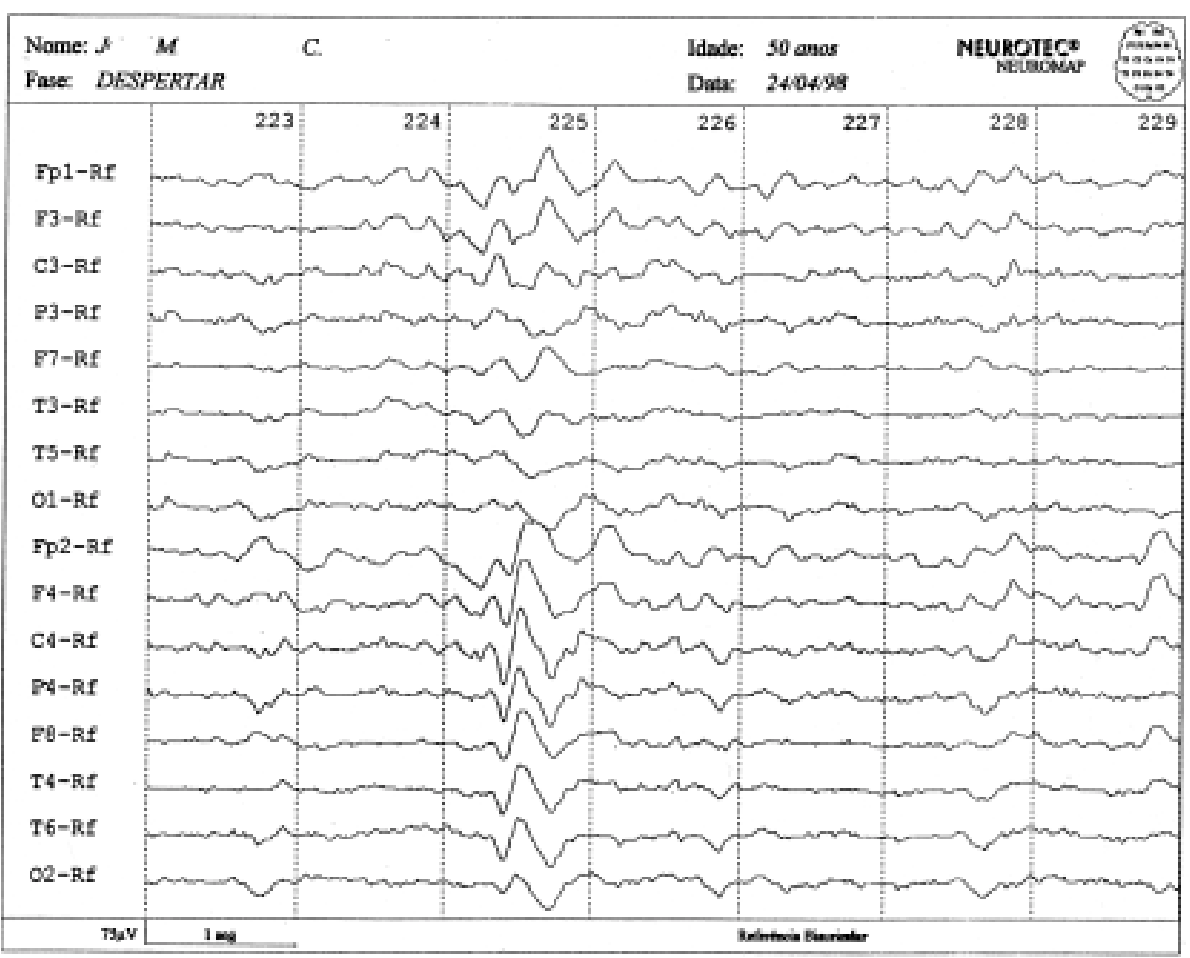

FIGURA 6

O foco da fig. 5, no mesmo instante, ao ser visto com a montagem biauricular, é melhor evidenciado à direita. região central, temporal média e occipital. Entretanto, a identificação de AEF na região frontal e/ou temporal $^{4,5}$, no EEG desses pacientes, pode gerar dificuldade diagnóstica: ausência? epilepsia frontal? epilepsia do lobo temporal? O EEG em sono, assim como as características semiológicas da crise e a reposta ao tratamento instituído, podem ajudar no diagnóstico diferencial.

\section{Epilepsia rolândica}

Uma das epilepsias mais comuns da criança e do adolescente, benigna, em que, em torno dos 16-18 anos de idade as crises parciais motoras desaparecem. O EEG apresenta atividade elétrica cerebral de base normal e AEF na região central ou temporal média (Fig. 9), sobretudo durante o sono, quando fica muito freqüente. A identificação do dipólo nessa epilepsia é um dos importantes critérios para considerá-la como benigna $^{6,7}$.

\section{Epilepsia do lobo temporal}

As crises parciais complexas da epilepsia do lobo temporal são as crises mais freqüentes do adulto e geralmente rebeldes ao tratamento, sendo a lobectomia temporal um dos recursos terapêuticos, com excelentes resultados na maioria dos casos. O EEG apresenta AEF na região temporal anterior (Fig. 10). O sono é importante nesses tipos de pacientes, principalmente quando o EEG é normal durante a vigília. Devemos lembrar que a AEF pode ser por onda aguda, complexo pontaonda, polipontas ou por onda lenta ${ }^{8}$.

\section{Síndrome de West}

Os espasmos infantis nas crianças entre 3 meses e 1 ano de idade, 


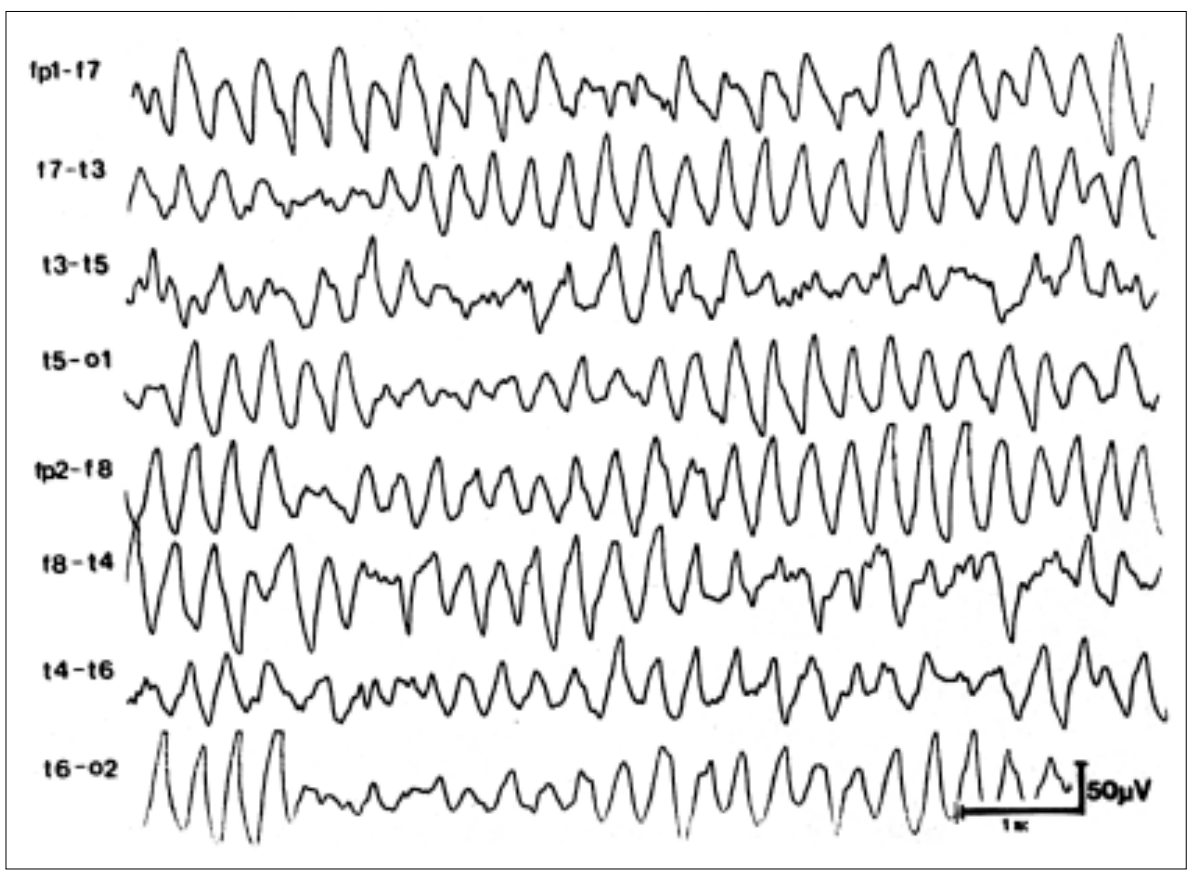

FIGURA 7

Ondas lentas difusas e bilaterais, durante a hiperpnéia.

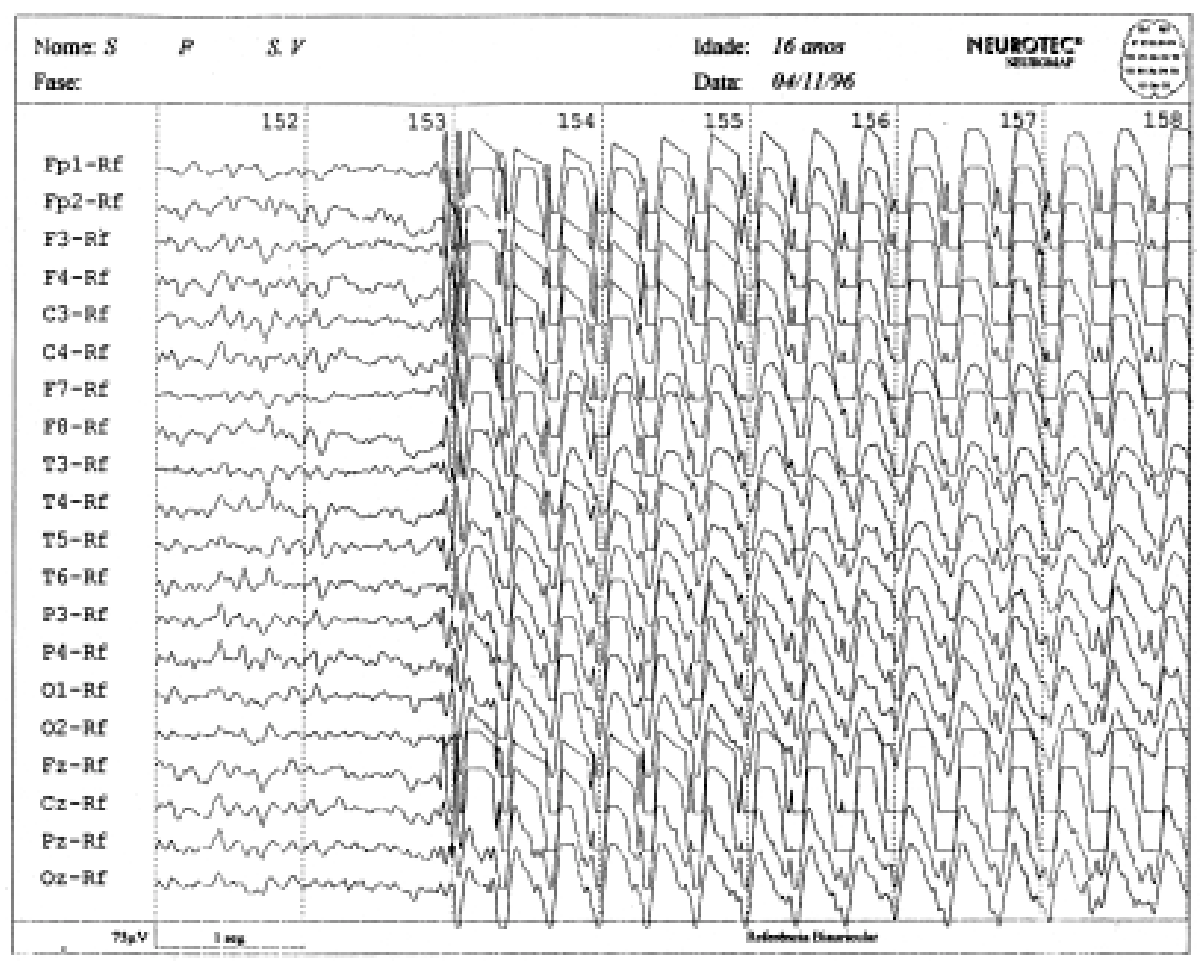

FIGURA 8

Complexos ponta-onda $3 \mathrm{~Hz}$ difusos e bilaterais da crise de ausência. não devem ser sempre encarados como a síndrome de West (SW). É necessário que o EEG apresente a hipsarritmia, isso ajuda a estabelecer o diagnóstico diferencial com espasmos infantis que não são enquadrados como SW. São vários os quadros que simulam $\mathrm{SW}$, porém o EEG é fundamental nessa distinção. Além disso, o EEG é importante na SW criptogênica, podendo fazer a diferenciação se estamos diante de uma SW benigna (primária) ou secundária ${ }^{9}$.

\section{Síndrome de Lennox- Gastaut}

A síndrome de Lennox-Gastaut (SLG), encefalopatia epiléptica grave, com retardo mental, que geralmente inicia em torno dos 2 anos de idade, sobretudo com crises de ausência atípica, crises tônicas e drop attacks, apresenta no EEG atividade de base alentecida e complexos ponta e poliponta-onda, 1-2 $\mathrm{Hz}$, difusos e bilaterais, de predomínio frontal, semicontínuos durante o sono, e polipontas. O EEG ajuda a estabelecer o diagnóstico diferencial com outras epilepsias que simulam a SLG, os chamados pseudo-SLG.

\section{Terminologia}

Devemos evitar o uso de termos como disritmia, atividade irritativa, equivalente epiléptico e foco irritativo. Temos preferência por "atividade epileptiforme caracterizada por ondas agudas, ondas lentas, complexos ponta-onda ou polipontaonda, polipontas de projeção na região ...". As ondas agudas e ondas lentas geralmente são AEF, enquanto os complexos ponta ou polipontaonda e polipontas são descritos freqüentemente como atividade epileptiforme difusa e bilateral, pois são encontrados nas epilepsias 


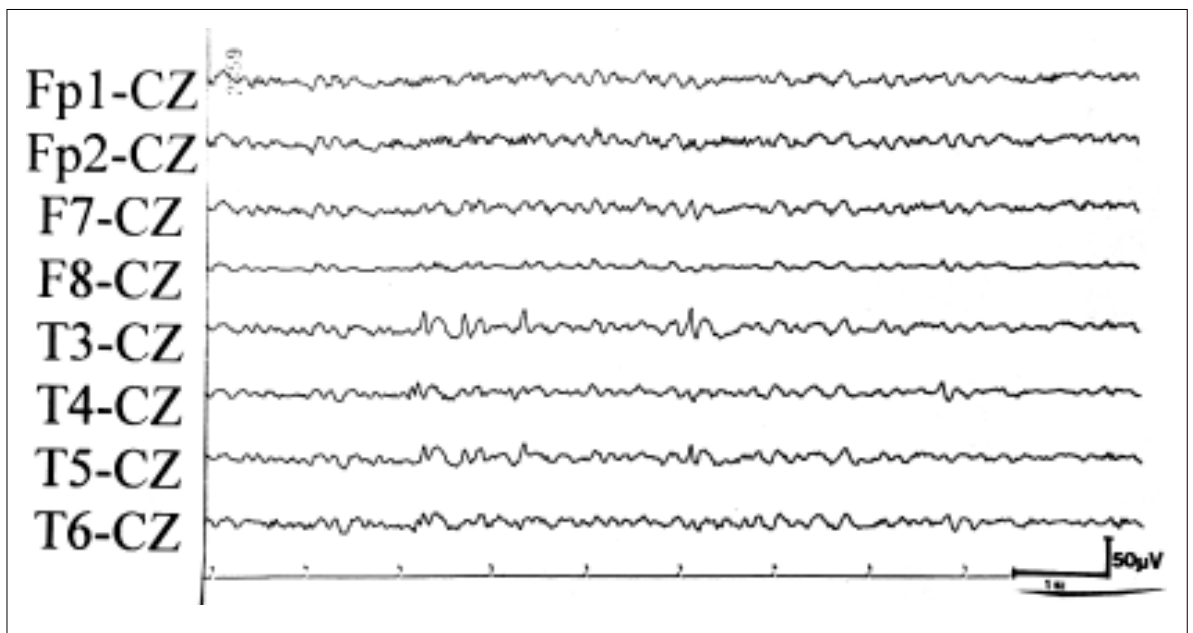

FIGURA 9

Foco temporal médio esquerdo (rolândico).

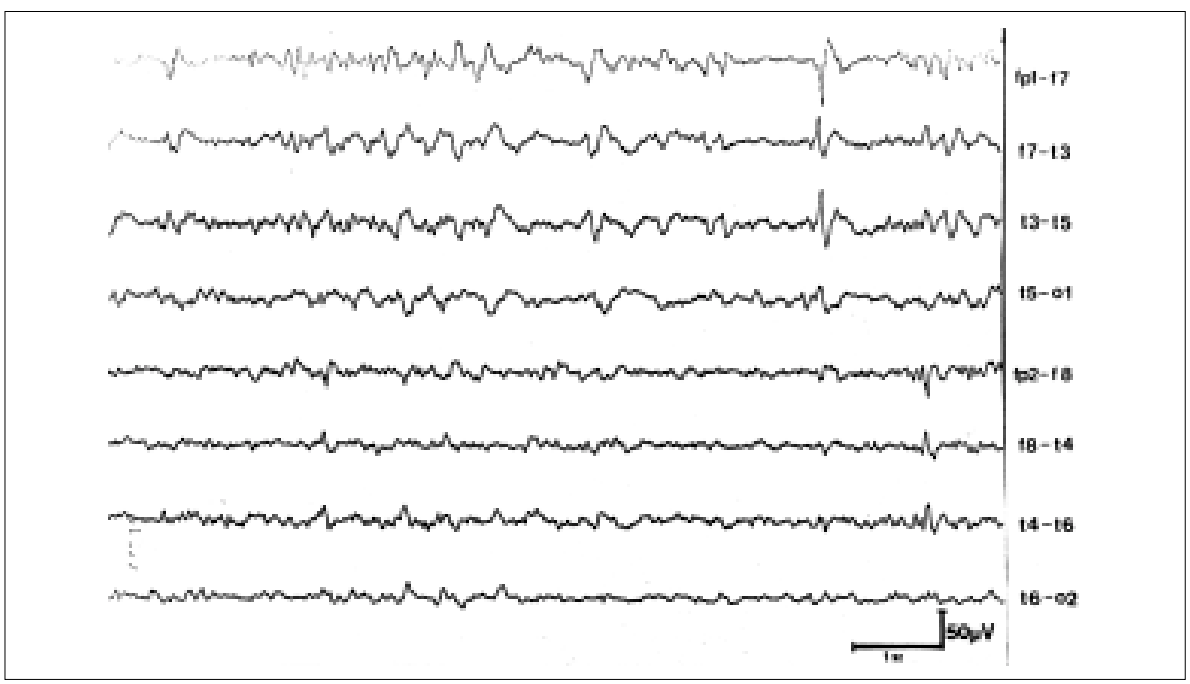

FIGURA 10

Foco temporal anterior bilateral, mais evidente à esquerda.

conseqüência não deve ser estabelecido um tratamento com drogas antiepilépticas. Parentes de pacientes epilépticos, pacientes com diagnóstico de enxaqueca, crianças hiperativas, distúrbio escolar, dor abdominal, perda de folego e crianças normais podem apresentar alterações no EEG e sem história de crise epiléptica ${ }^{10}$. Do mesmo modo, pacientes idosos comumente apresentam ondas agudas e ondas lentas na região temporal e, também, não devem ser catalogados de epilépticos ${ }^{11}$. Contudo, achamos que a presença de AEF de projeção frontal ou temporal anterior mereça uma investigação e questionamento em relação à ocorrência de "desligamentos" e "esquecimentos" (crises parciais complexas), mesmo que o paciente negue crises, pois essa AEF geralmente é sintomática e lesional (epilepsia parcial secundária).

\section{“Status E pi lepti cus"}

Pode haver dificuldade diagnóstica no status epilepticus (SE) não-convulsivo, seja por crise parcial complexa ou por ausência. Esses pacientes, às vezes, são diagnosticados como portadores de doença psiquiátrica, pois suas formas de apresentação são muito variadas, desde uma confusão mental até um distúrbio de conduta. Nesses casos, o EEG é imprescindível para o

generalizadas. Entretanto, devemos lembrar que podemos também identificar esses padrões nos EEG das epilepsias parciais. Nesses casos, o achado de AE caracterizada por complexo ponta-onda ou polipontas focais, e que se propagam para regiões ipsi e contralaterais, pode dar ao EEG um aspecto difuso e bilateral (pseudogeneralizado).

\section{Atividade epil eptiforme no EE G x paciente sem epilepsia}

A identificação no EEG de AEF ou difusa e bilateral não significa que o paciente seja epiléptico e por diagnóstico diferencial. No SE que se apresenta apenas como afasia ${ }^{12}$ ou sob a forma de hemiparesia ${ }^{13}$, o EEG afasta o diagnóstico de AVC e confirma essa forma incomum de SE. Do mesmo modo, alguns casos de SE tipo parcial simples não devem ser confundidos com crises de enxaqueca ${ }^{14}$.

Em algumas síndromes a atividade epileptiforme contínua ou semicontínua não deve ser interpretada como SE. A identificação desses quadros evita condutas emergenciais. Por exemplo, a síndrome da ponta-onda contínua do sono não-REM ("status" eletrográfico do sono não-REM) não deve ser confundida com SE não convulsivo, pois ocorre apenas no sono não-REM e desaparece com o despertar e durante o sono REM ${ }^{15}$ 


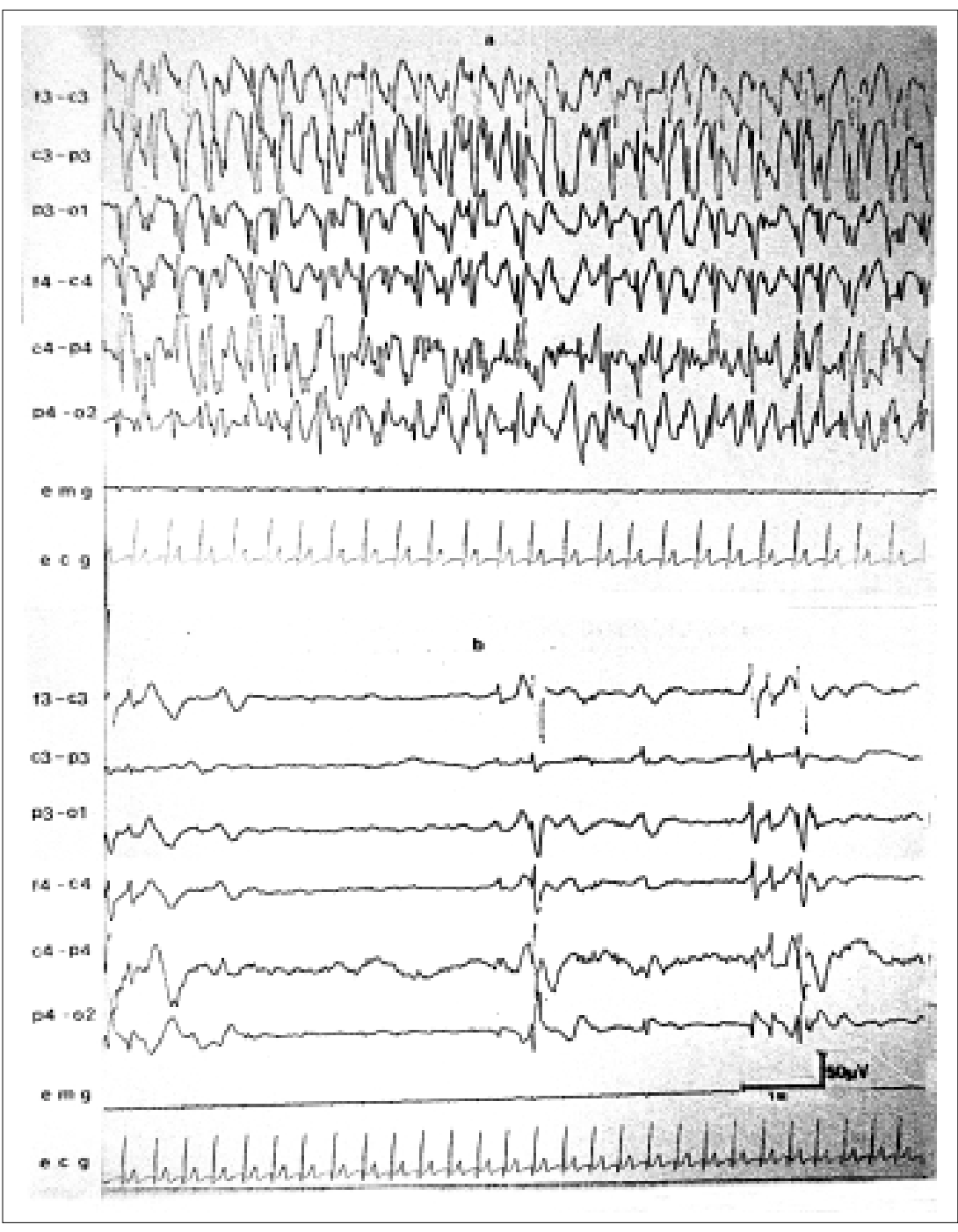

FIGURA 11

"Status" eletrográfico do sono não-REM (a) desaparece durante o REM (b).

\section{EEG X DEMÊNCIAS}

$\mathrm{Na}$ demência mais freqüentemente diagnosticada, a de Alzheimer (DA), o EEG na fase inicial pode ser útil no diagnóstico diferencial com uma depressão (pseudodemência), pois uma redução na freqüência do ritmo alfa em EEG seriados sugere que não seja apenas uma depressão, mas sim a fase inicial da $\mathrm{DA}^{20}$. Alguns pacientes com DA podem apresentar no EEG um padrão periódico curto e difuso, semelhante ao da demência de Creutzfeldt-Jakob (CJ $)^{21,22}$, que pode gerar dificuldade diagnóstica, sobretudo se esses pacientes com DA apresentarem mioclonias.

A demência de CJ caracteriza-se no EEG por um padrão periódico curto e difuso, com ondas trifásicas de predomínio anterior. Entretanto, esse padrão periódico pode predominar nas regiões posteriores ou apresentar-se no EEG como periódico curto e focal ("PLEDs") ${ }^{23}$. Devemos lembrar que o padrão periódico na demência de CJ nem sempre está presente, mesmo com EEG seriados durante a evolução da doença. Quando isso ocorre em pacientes com CJ, sobretudo na forma familiar, na faixa etária dos 50-65 anos de idade e que não apresentam mioclonias, pode ser difícil um diagnóstico diferencial com DA, dependendo da fase em que se encontra o paciente ${ }^{24,25}$. O diagnóstico diferencial com a DA também pode ser dificultado quando a demência de CJ se apresenta apenas com afasia progressiva, sem mioclonias e sem padrão periódico no EEG (apenas na fase final), em paciente na faixa

(fig.11). Esse achado no EEG da ponta-onda contínua também pode ocorrer durante a vigília e é conhecido como "status" eletrográfico da vigília ${ }^{16}$. A epilepsia com ponta-onda occipital reativa a abertura e fechamento ocular, em que a atividade epileptiforme occipital contínua, com os olhos fechados, desaparece com a abertura ocular ${ }^{17}$. Algumas disgenesias corticais também cursam com atividade epileptiforme contínua, porém sem SE clínico. Por outro lado, durante as crises parciais simples geralmente o EEG cursa sem alteração ${ }^{18}$, o que não deve ser interpretado como pseudocrise ou doença extrapiramidal. Nesses casos, o aumento do número de eletrodos facilita o registro da crise no $E_{E G}{ }^{19}$. etária dos 60 anos $^{26}$. Algumas drogas, como o baclofen e o carbonato de lítio, podem provocar no EEG o padrão periódico curto e difuso e o paciente clinicamente estar demente, simulando uma demência de $\mathrm{CJ}^{27,28}$. Nesses casos, a retirada de droga leva ao desaparecimento da periodicidade e da demência.

A demência da coréia de Huntington apresenta no EEG um padrão de atenuação da atividade elétrica cerebral, difuso e bilateral. Na demência por múltiplos infartos, as alterações são geralmente multifocais com paroxismos por ondas lentas theta e delta. $\mathrm{Na}$ doença de Parkinson que cursa com demência, o EEG apresenta alentecimento difuso e bilateral ${ }^{29}$. 


\section{ENCEFALOPATIAS METABÓLICAS}

A encefalopatia metabólica (EM) hepática apresenta no EEG alentecimento difuso e bilateral, com predomínio de ondas theta e/ou delta, assim como períodos de supressão, dependendo da gravidade da encefalopatia. A presença de ondas trifásicas típicas, de predomínio anterior, é um achado no EEG muito comum nesse tipo de EM. A EM renal apresenta achados semelhantes aos da hepática, porém não é comum a ocorrência de ondas trifásicas típicas e mais freqüentemente o EEG apresenta crises eletrográficas, sobretudo durante a fotoestimulação. Na hipocalcemia o EEG pode apresentar atividade epileptiforme freqüente, por vezes contínuas, sob forma de status epilepticus, de predomínio posterior ou difusas e bilaterais.

\section{VARIANTES BENIGNAS DO EEG}

A identificação de alguns padrões eletrográficos conhecidos como variantes benignas (VB) é importante para que o EEG não seja laudado como anormal. Algumas VB são semelhantes às atividades epileptiformes, como o complexo ponta-onda $6 \mathrm{~Hz}$ (ponta-onda "fantasma"), a pequena ponta benigna do sono, a pseudo ponta-onda e as wickets spikes. Temos também as pontas positivas 6/ $14 \mathrm{~Hz}$, o "SREDA" (Fig. 12) ${ }^{30}$, que pode ser confundido com crise eletrográfica, a descarga rítmica temporal média, a pseudo-ponta-onda (hipersincronia hipnagógica), o ritmo da fenda em pacientes craniotomizados e mais recentemente o potencial "N" ${ }^{31}$. No caso de dúvida entre a atividade epileptiforme e a VB, a primeira geralmente fica mais freqüente conforme o sono vai aprofundando, enquanto a VB só aparece durante a sonolência ${ }^{32}$.

\section{RECÉM-NASCIDO}

O EEG realizado no recémnascido (RN) é um dos exames mais importantes da investigação neurológica, sobretudo pelo aspecto prognóstico e, às vezes, como imprescindível para o diagnóstico de determinados quadros clínicos que atingem o RN. No RN, além do EEG, também monitoramos os movimentos oculares, a respiração, outro eletrodo para EMG e um para o ECG.
Alguns padrões eletrográficos significam mau prognóstico, ou seja, o risco é alto para que o RN apresente uma encefalopatia: a) as pontas positivas freqüentes ${ }^{33}$; b) surto-supressão; c) traçado de baixa amplitude; d) longos períodos de inatividade (> 30s, no prematuro), com ausência de grafoelementos próprios da prematuridade (delta brush; sawtooth). Ao contrário, o EEG normal do prematuro (traçado descontínuo, com período de atenuação variando de 5 a 20 s; presença de delta brush e de sawtooth) ou do RN a termo (tracé alternant) correlacionam-se com um bom prognóstico, tanto do ponto de vista motor como intelectual.

Em relação ao diagnóstico, o EEG é importante para complementar o diagnóstico da encefalopatia de Ohtahara, a encefalopatia mioclônica precoce de Aicardi, pelo aspecto de surto-supressão. No status convulsivo de $5^{\circ}$ dia, a presença do padrão eletrográfico theta pointu alternant complementa o diagnóstico. Além disso, o EEG é imprescindível para o diagnóstico de determinados tipos de crises epilépticas neonatais, como apnéia, taquipnéia, mastigação, piscamentos e outros tipos de crises sutis ou ocultas do RN.

\section{COMAS}

O EEG tem importância prognóstica no coma traumático, pois a identificação do spindle coma ou sleep like e a presença de reatividade do EEG aos estímulos dolorosos, na fase aguda do coma, correlacionam-se a um melhor prognóstico em $87 \%$ dos casos, enquanto a ausência desse achados, com atividade delta difuso e sem reatividade no EEG à estimulação dolorosa, está associada à má evolução em $86 \%$ dos pacientes comatosos ${ }^{34}$.

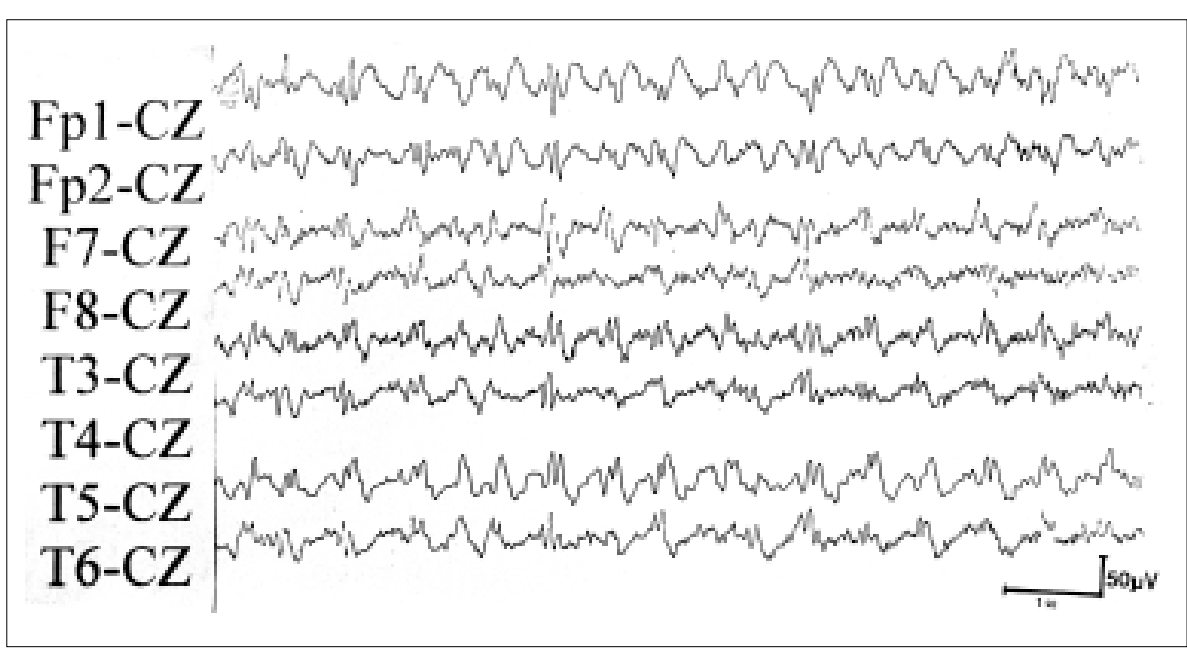

FIGURA 10

Foco temporal anterior bilateral, mais evidente à esquerda. 
A presença da atividade alfa em um paciente comatoso leva ao diagnóstico do "coma alfa". Esse achado no EEG ocorre geralmente em pacientes com lesão de tronco cerebral, sobretudo na região pontina, e as etiologias mais comuns são o AVC e trauma ${ }^{35}$.

Alguns pacientes comatosos que não melhoram, apesar de todos os exames neurorradiológicos e clínicos gerais sugerirem que o paciente está com boa evolução, podem estar em SE não-convulsivo. O EEG nesses casos é fundamental, pois ao demonstrar o status eletrográfico, apesar de clinicamente não ser visível, está indicado o tratamento de emergência com drogas antiepilépticas, o que leva o paciente a uma melhor e rápida recuperação.

Em relação à morte cerebral, para considerarmos o EEG com silêncio elétrico cerebral, devemos observar se há presença de hipotermia, uso de drogas (benzodiazepínicos, barbitúricos) e alterações metabólicas. Devemos evitar falar em morte cerebral para o recémnascido até 7 dias de vida, pois existem muitas discordâncias entre o quadro de coma, angiografia cerebral e resultado do EEG, devido à imaturidade do sistema nervoso central. Até os 2 anos de idade é recomendável dois exames clínicos e 2 exames de EEG, com intervalo variando de 24 a $48 \mathrm{~h}$. O exame deve ser realizado por 30 minutos, com todos os eletrodos cerebrais colocados de acordo com sistema 10-20, aumentar a sensibilidade para $2 \mu \mathrm{V} / \mathrm{mm}$, usar filtros de $0,5-70 \mathrm{~Hz}$ e não deve ser observada reatividade no EEG aos estímulos dolorosos, visuais e auditivos. Em relação à colocação dos eletrodos no couro cabeludo, devemos usar distâncias duplas, com um mínimo de $10 \mathrm{~cm}$ de separação intereletrodos: F3P3; C3-O1; FP1-T3; F7-T5; F4-P4; C4-O2; FP2-T4; F8T6. Do mesmo modo, aplicamos essa medida para as montagens transversas anterior (F7-Fz; Fz-F8), média (T3-Cz; Cz-T4) e posterior (T5-Pz; Pz-T6). Devemos deixar um canal para o registro dos potenciais cardíacos e outro para a monitorização do ambiente (eletrodos podem ser colocados sobre o dorso da mão) ${ }^{36}$.

\section{EEG CONVENCIONAL X EEG DIGITAL $X$ MAPEAMENTO CEREBRAL}

Temos observado muita dúvida em relação à importância do EEG digital (ED) e o mapeamento cerebral (MC), assim como acharem que o ED e o MC são sinônimos. Podemos dizer que EEG convencional é o registro que não utiliza o computador, não podendo ser arquivado em disquetes, não permitindo a mudança das montagens após a captação do registro e nos obriga a utilização de pilhas de papel. Ao contrário, o EEG digital é exibido na tela do monitor colorido, após ser processado pelo microcomputador, podendo ser armazenado em disquetes, dispensando o uso de arquivos gigantes para empilhamento de papéis. O ED possui grande vantagem em relação ao EC, por permitir o manuseio das montagens após o registro, de grande importância no estudo das atividades epileptiformes. O MC é a representação gráfica do ED por meio de mapas elaborados pelo microcomputador ${ }^{36}$. Encerrado o registro do ED, realiza-se a leitura e seleciona-se os melhores momentos para o MC, ou seja, este só é realizado após a escolha dos fragmentos do ED. Dessa forma, é fundamental a correta seleção dos melhores momentos, pois todos os passos seguintes durante a realização do MC serão baseados nos fragmentos escolhidos. Uma escolha inadequada pode levar a uma interpretação e conclusão errôneas do exame.

Achamos que brevemente o ED dominará o cenário mundial da eletrencefalografia, de modo que os aparelhos de EEG convencional farão parte de um passado da história do EEG. Em relação ao MC, que é uma extensão do ED, não deve ser interpretado isoladamente, mas em conjunto com o ED. Somos de opinião que o MC é um exame que não deve ser solicitado como rotina, estando reservado apenas para a pesquisa científica em alguns campos na neurologia e psiquiatria.

\section{SUMMARY}

General and Practical Aspects of the EEG

The knowledgment of some practical aspects in the analysis of the EEG are important to avoid misdiagnosis in the final reports. About epilepsies, the correct use of the montages makes easy the study of the EEG and we can make the diagnosis of epileptic syndromes. In demented patients we may find different patterns without a patognomonical type. Benign variants of the EEG must not be misinterpreted as abnormal findings. In the same way, patients with epileptiform activity on EEG without a history of epilepsy, should not receive antiepileptic drugs. The EEG in the newborn has diagnostic and prognostic values in the encephalic lesions. In non-convulsive epileptic "status" the EEG makes the differential diagnosis. In metabolic encephalopathies we can evaluate the degree of severity by the EEG. The comas may have their prognosis established by the EEG and it helps in the diagnosis of brain death. The brain mapping, carried out only after the digital $E E G$, is not a routine exam.

\section{KEY WORDS}

EEG, general aspects.

\section{Referências}

1. Lima, M.M. Estudo dos focos da linha média em uma população. São Paulo, 1997, 111 pg. Tese - Mestrado - Escola Paulista de Medicina - UNIFESP.

2. Jasper, H. The ten-twenty electrode system of the International Federation. Electroenceph clin Neurophysiol, 10:371-5, 1958.

3. Silva, D.F.; Lima, M.M.; Anghinah, R.;Zanoteli, E.; Lima, J.G.C. Atypical EEG pattern in children with absence seizures. Arq Neuropsiquiatr, 53:258-61, 1995.

4. Lombroso, C.T. Consistent EEG focalities detected in subjects with primary generalized epilepsies monitored for two 
decades. Epilepsia, 38:797-812, 1997.

5. Kudo, T.; Sato, K.; Yagi, K.; Seino, M. Can absence status epilepticus be of frontal lobe origin? Acta Neurol Scand, 92:472-7, 1995.

6. Coelho, C.M.F. Estudo do dipólo rolândico em uma população. São Paulo, 1996, 120 pg. Tese - Mestrado - Escola Paulista de Medicina - UNIFESP.

7. Djabraian, A.A.F.; Coelho, C.M.F.; Pradella-Hallinan, M.; Silva, D.F. A study of dipole rolandic spikes in partial epilepsies of childhood. Epilepsia, 1997; 38(Suppl 3):119.

8. Normand, M.M.; Wszolek, Z.K.; Klass, D.W. Temporal intermittent rhythmic delta activity in electroencephalograms. $\mathrm{J}$ Clin Neurophysiol, 12:280-4, 1995.

9. Dulac, O.; Plouin, P.; Jambaqué, I. Predicting favorable outcome in idiopathic West Syndrome. Epilepsia, 34:747-56, 1993.

10. Lerman, P.; Kivity-Ephraim, S. Focal epileptic EEG discharges in children not suffering from clinical epilepsy: etiology, clinical significance, and management. Epilepsia, 22:551-8, 1981.

11. Asokan, G.; Pareja, J.; Niedermeyer, E. Temporal minor slow and sharp EEG activity and cerebrovascular disorder. Clin Electroenceph, 18:201-10, 1987.

12. Wells, C.R.; Labar, D.R.; Solomon, G.E. Aphasia as the sole manifestation of simple partial status epilepticus. Epilepsia, 33:84-7, 1992.

13. Hanson, P.A.; Chodos, R. Hemiparetic seizures. Neurology, 28:920-23, 1978 .

14. Walker, M.C.; Smith, J.M.; Sisodiya, S.M.; Shorvon, S.D. Case of simple partial status epilepticus in occipital lobe epilepsy misdiagnosed as migraine: clinical, electrophysiological, and magnetic resonance imaging characteristics. Electroenceph cli. Neurophysiol, 36:1233-6, 1995.

15. Djabraian, A.A.F.; Pradella-Hallinan, M.; Silva, D.F. Continuous spikes and waves during slow sleep: a study of 15 cases from Brazil. Epilepsia, 38(Suppl 3):121, 1997.

16. Gokyigit, A.; Caliskan, A. Diffuse spike-wave status of 9-year duration without behavioral change or intellectual decline. Epilepsia, 36:210-3, 1995.

17. Gastaut, H. Benign epilepsy of childhood with occipital paroxysms. In: Roger, J.; Dravet, C.; Bureau, M.; Dreifuss, F.E.; Wolf, P., ed. Epileptic Syndromes in Infancy, Childhood and Adolescence. John Libbey \& Company Ltd., London, 1., 1985, pp. 159-70.

18. Devinsky, O.; Kelley, K.; Porter, R.J.; Theodore, W.H. Clinical and electroencephalographic features of simple partial seizures. Neurology, 38:1347-52, 1988.

19. Bare, M.A.; Burnstine, T.H.; Fisher, R.S.; Lesser, R.P. Electroencephalographic changes during simple partial seizures. Epilepsia, 35:715-20, 1994.

20. Prinz, P.N.; Vitiello, M.V. Dominant occipital (alpha) rhythm frequency in early stage Alzheimer's disease and depression. Electroenceph clin Neurophysiol, 73:427-32, 1989.

21. Ehle, A.L.; Johnson, P.C. Rapidly evolving EEG changes in a case of Alzheimer disease. Ann Neurol, 1:593-5, 1977.

22. Watson, C.P. Clinical similarity of Alzheimer and CreutzfeldtJakob disease. Ann Neurol, 6:368-9, 1979.
23. Furlan, A.J.; Henry, C.E.; Sweeney, P.J.; Mitsumoto, H. Focal EEG abnormalities in Heidenhain's variant of JakobCreutzfeldt disease. Arch Neurol, 38:312-4, 1981.

24. Tietjen, G.E.; Drury, I. Familial Creutzfeldt-Jakob disease without periodic EEG acticity. Ann Neurol, 28:585-8, 1990.

25. Brechet, R.; Sicard, C.; Moret-Chalmin, C.; Olivesi, L.; Cathala, F.; Brown, P.; Cathala, H.P. Étude électro-encéphalographique de vingt-cinq cas de maladie de Creutzfeldt-Jakob. Rev. EEG Neurophysiol., 10:55-63, 1980.

26. Mandell, A.M.; Alexander, M.P.; Carpenter, S. CreutzfeldtJakob disease presenting as isolated aphasia. Neurology, 39:55-8, 1989.

27. Hormes, J.T.; Benarroch, E.E.; Rodrigues, M.; Klass, D.W. Periodic sharp waves in baclofen-induced encephalopathy. Arch Neurol, 45:814-5, 1988.

28. Smith, S.J.M.; Kocen, R.S. A Creutzfeldt-Jakob like syndrome due to lithium toxicity. J Neurol Neurosurg Psych, 51:120-23, 1988.

29. Soikkeli, R.; Partanen, J.; Soininen, H.; Paakkonen, A.; Riekkinen Sr., P. Slowing of EEG in Parkinson' s disease. Electroenceph Clin Neurophysiol, 79:159-165, 1991.

30. Silva, D.F.; Zanoteli, E.; Lima, M.M.; Anghinah, R.; Lima, J.G.C. Subclinical rhythmic electrographic discharge of adult: an atypical evolution. Arq Neuropsiquiatr, 53:266-9, 1995.

31. Silva, D.F.; Lima, M.M.; Kanda, P.M.; Anghinah, R.; Zanoteli, E.; Lima, J.G.C. Atypical pattern related to $14 \mathrm{~Hz}$ positive spikes. Arq Neuropsiquiatr, 53:262-5, 1995.

32. Silva, D.F.; Lima, M.M.; Anghinah, R. Variantes benignas do eletrencefalograma. Rev Neurociências, 2:14-8, 1994.

33. Marret, S.; Parain, D.; Jeannot, E.; Eurin, D.; Fessard, C. Positive rolandic sharp waves in the EEG of the premature newborn: a five year prospective study. Arch Dis Child, 67:948$51,1992$.

34. Bricolo, A.; Turella, G. Electroencephalographic patterns of acute traumatic coma: diagnostic and prognostic value. J Neurosurg Sci, 17:278-85, 1973.

35. Chatrian, G.E.; Lowell, M.D.; White, E. Jr.; Shaw, C.M. EEG pattern resembling wakefulness in unresponsive decerebrate state following traumatic brain stem infarct. Electroenceph Clin Neurophysiol, 16:285-9, 1964.

36. American Electroencephalographic Society Committee. Guideline three: minimum technical standards for EEG recording in suspected cerebral death. J Clin Neurophysiol, 3(Suppl. 1):12-7, 1986.

37. Silva, D.F.; Lima, M.M.; Anghinah, R.; Lima, J.G.C. Mapeamento Cerebral. Rev. Neurociências, 3:11-18, 1995.

\section{Endereço para correspondência:}

Délrio Façanha da Silva

Universidade Federal de São Paulo

Escola Paulista de Medicina - Disciplina de Neurologia

Rua Botucatu, 740 - Vila Clementino

CEP 04023-900 São Paulo (SP)

E-mail: thierry@internetcom.com.br 